\title{
PRINCÍPIOS PRÁTICOS ATUAIS DA HORMÔNOTERAPIA (*)
}

\author{
ATTILIO Z. FLOSI \\ Academico Interno da 1.a M. H. (Serviço do Prof. Almeida \\ Prado). Auxiliar efetivo da Secção de Enıdocrinologia (S. do Dr. \\ Mesquita Sampaio) do Ambulatório de Neurologia da Santa Casa
}

$\mathrm{O}$ evoluir constante da endocrinologia tem modificado os princípios gerais da terapêutica hormônal. Com efeito, antigamente empregavam-se com exclusividade os "extratos glandulares", cuja atividade terapêutica é, muita vez precaria, sendo ainda controlada com dificuldades. Posteriormente, numerosas pesquizas, principalmente experimentais, vieram demonstrar a existência nesses extratos de vários princípios hormônicos com atividade biológica específica. Consequêntemente, passou-se a emprega-los isoladamente, de acôrdo com as suas atividades fisiológicas, obtendo-se melhores resultados. Por outro lado, os progresso da bio-quimica tornaram possivel o conhecimento da estrutura molecular de vários hormônios, chegando-se mesmo a prepará-los sintéticamente, o que veio facilitar o seu emprego ná prática, tornando-os mais ativos e de preço menos elevado.

$$
* * *
$$

Atualmente o numero de hormônios descrito é enorme. No entanto, já se observa entre os endocrinologistas modernos uma reação contra o espirito analítico de seus predecessores, admitindo-se a pólivalencia funcional de um único hormonio em função da via de administração, dóse, técnica de extração, etc.

A via de administração dos hormônios depende de sua estrutura molecular. Assim, os que são de natureza proteica, como a insulina, os hormônios hipofisarios e o paratormônio não tem ação quando empregados por via oral, pois são inativados pelos sucos digestivos que os destróem. Outros, como a tiroxina (exceção à regra) a estrina e a metil-testosterona são resistentes aos fermentos digestivos, portanto podem ser empregados por tal via. Neste grupo poder-se-á incluir a pregneninolona que é uma substância capaz de exer-

(*) Palestra realizada no Departamento Científico do C. A. O. C. em 25 de Julho de 1941. 
cer ação semelhante a da progesterona que, como sabemos, é inativa por via oral.

Atualmente a maioria dos hormônios é empregada sob a forma de injjeções sub-cutaneas ou intra-musculares, usando-se de preferencia veículo oleoso. No entanto, em determinados casos, alguns. hormônios (estrina, testosterona, etc) pódem ser empregados sob a. forma de unções, pois são perfeitamente absorvidos por via cutânea.

No tratamento sintomático do diabete insipidus emprega-se com: bons resultados preparações recentes de extrato de lóbo posterior da hipófise por via nasal.

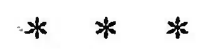

Verificou-se que a atividade dos hormômios esterólicos é inversamente proporcional à velocidade de sua absorção. Baseando-se neste importante principio de hormônoterapia, procurou-se aumentar a ação terapêutica dos hormônios aplicando-os sob a forma de tabletes em implantações subcutâneas reduzindo-se, deste modo, a velocidade de absorção. Thales Martins, (1) confirmando os trabalhos de Deansley e Parkes, observou que a implantação subcutânea de $2 \mathrm{mgr}$. de testosterona exercia maior ação que duas injeções de 5 . miligramas sôbre a contratibilidade da genitalia acessória masculina. Recentemente THORN e colaboradores (2) empregaram a desoxicorticosterona segundo esse método com resultados satisfatórios, verificando que uma unica tablete de hormônio era suficiente para manter o organismo durante meses sob a sua ação. Aliás, os hormônios. esteróliticos, que apresentam constituição química bem definida, foram os únicos que até agora deram resultados quando aplicados segundo. tal método que apresenta as vantagens de permitir um menor numero de aplicações, com efeitos ainda mais enérgicos. Sabemos que certos hormônios (testoterona, estradiol) quando esterificados tornamse mais ativos. Tal efeito seria em parte devido à redução da velocidade de sua absorçã̃o. (Thales Martins) (3).

$$
* * *
$$

A observação demonstra que o emprego repetido de certos hormônios produz, depois de certo tempo, no organismo que está sob a sua ação, um estado de refratariedade que seria devido à formação de substâncias inhibidoras dos hormônios, denominadas antihormônios. BAUER (4) injetando pequenas doses de tiroxina em. cobaia verificou posteriormente que ela se tornára resistente a tal droga, acreditando-se na formação de uma substância anti-tiroxinica. Numerosas outras experiências confirmaram este fenômeno. São particularmente interessantes os trabalhos de PARkes e Rowlands (5). que obtiveram estado de refratariedade em cobaia normal frente aos: 
hormonios gonadotrópicos hipofisários, injetando-lhes sôro de cobaia préviamente tratada pelos hormônios referidos.

Collip (6) admite a existência deses anti-hormonios normalmente 'no organismo. Entretanto, tal hipótese não é mais aceita, acreditando-se que somente os hormônios impurificados por proteinas extranhas são capazes de formá-los. Segundo Gordon (7) e colaboradores essas substâncias são elaboradas pelas células do sistema retículo-endotelial. Devemos pois, ter o cuidado de empregar somente os hormônios purificados, afim de evitar a resistência do organismo. Esta teoria explica porque, muitas vezes, após a aplicação continuada de certos hormônios eles se tornam inativos.

Após essa análise sucinta de alguns princípios gerais de hormônoterapia, podemos indicar as bases fundamentais do emprego dos principais hormônios. Limitar-nos-emos a assinalar apenas os que apresentam, na atualidade ,importância prática. Consequentemerite, não serão aqui estudados os hormônios tímicos, epifisários certos hormônios hipofisários (metabólicos, adrenotrópicos etc.). Por outro lado, não será analisado o tratamento das diferentes endocrínopatias, mas apenas indicaremos genericamente o possivel contingente terapêutico glandular.

\section{HIPÓFISE ANTERIOR}

Hormônio de crescimento - A experimentação demonstra de maneira inconteste a influência da hipófise sôbre o crescimento. Assim, a hipófisectomia praticada em animais jovens provoca-lhes parada do crescimento (AsCHNER, 1909). Administrando-se extratos hipofisários aos animais hipófisectomisados não sómente se lhes restabelece o crescimento como poder-se-á provocar-lhes o gigantismo. (Evan e Long; 1921).

Segundo alguns autores a hipófise age sôbre o crescimento por intermédio dos "target-organ hormones", isto é hormonios tireotrópico, adrenotrópico, gonadotrópico e lactogênico. No entanto, segundo Evans, (8) pode-se restabelecer o crescimento de um animal jovem hipófisectomisado empregando-se extratos hipofisários isentos desses hormônios. Admite-se pois a existência de um hormônio com ação específica sôbre o crescimento, sendo denominado hormônio de crescimento ou somatotrópico Êle é elaborado pelas células eosinófilas encontradas no lóbo anterior da hipófise. $\mathrm{Na}$ clínica emprega-se tal hormônio com êxito no tratamento das perturbações do crescimento de origem hipofisária. Entretanto, antes de se instituir essa terapêtutica, faz-se mistér determinar precisamente a causa do "deficit hipofisário", tendo-se o cuidado de excluir a eventual presença de um tumor da hipófise, que requer tratamento especializado, (Radioterapia, cirurgica, posteriormente hormônoterapia). 
Para isso, além do exame clínico detalhado, torna-se necessário radiografias da séla turcida, exames neuro-ocular, eventuais vèntriculografias, exame do L. C. R., etc. Afastada a hipótese de um tumor, devemos verificar radiologicamente o estado dos ossos, pois sómente se obtem resultados quando ainda não houve soldadura das epifises, condição sine qua non. $\mathrm{Na}$ prática as radiografias da mão e do punho já nos pódem dar valiósas indicações. O hormônio de creșcimento é muito instavel, altera-se facilmente. Dever-se-á portanto empregar preparações recentes e isentas da fração gonadotrópica, que iria, caso contrário, apressar a maturidade sexual, determinando em consequência, a soldadura das epifises. Autores de grande experiência como Schaffer e Engelbach (9) têm obtido excelentes resultados na prática empregando o hormônio de crescimento no tratamento do nanismo hipofisário.

Estandardização - E' feita mediante ensaios biológicos em ratos. A unidade rata (U. R.) representa a menor quantidade diária de substância que injetada em rata adulta provoca-lhe aumento nítido de peso (10\%) no fim do periodo de 10 dias de ensáio.

\section{HORMONNIO LACTOGÊNICO}

Durante a gravidez a estrina (Fig. n. ${ }^{0} 3,11$ ) provoca o desenvolvimento da glândula mamária e dos canais galactóferos. Associação à progesterona (Fig. 3, 12) determina um desenvolvimento dos alveolos. No parto, a hipófise anterior livra-se da ação inhibidora da estrina lançando à circulação o hormônio lactogênico ou prolactina ou mamotropina que vai condicionar a secreção lactea. (Fig. n. $.^{\circ}, 5$ ).

As pesquisas de Seyle e Mac Koewn (10) demonstraram que a secreção desse hormônio sofre tambem a influência do sistema nervoso, pois a sucção estimula a sua produção. Segundo Collip (11) e colaboradores, além da estrina a repleção do útero inibe tambem a formação desse hormônio. Procurou-se empregá-lo na prática para estimular a secreção lactea das nutrizes, sendo ainda uma questão controvertida. Com efeito Kurzrok, Bates, Riddle e Müller (12) empregaram-no em nutrizes com secreção lactea deficiente, obtendo efeitos favoraveis. No entanto, os trabalhos recentes de STEWART e Pratt (13) não confirmaram esses resultados, pelo contrário, verificaram ser ineficaz a sua aplicação em clínica. Nutrizes que receberam mil U. P. diariamente durante 3 dias apresentaram um aumento de $40 \%$ da secreção lactea. Aumento maior (42\%), foi observado em nutrizes que receberam apenas uma solução salina pura.

O hormônio lactogênico é padronizado pelo test da secreção lactea do papo de pombo. Uma unidade internacional corresponde a atividade de $1 / 10$ de miligrama de um preparado estandard. 


\section{SUBSTANCIAS GONADOTRÓPICAS}

As substâncias gonadotrópicas constituem os estimulantes fundamentais das glândulas sexuais. São de origem hipofisária ou extra-hipofisária, de sorte que, tendo como fundamento a sua gênese, poder-se-á classificá-las em dois grandes grupos:

$10^{\circ}$ - Gonadotropínas hipofisárias - São constituidas por duas frações:

a) fração foliculinizante cuja ação é estimular a maturação dos folículos dos ovários e a ovulação ou o desenvolvimento do epitélio germinativo dos testículos. E' o hormônio de maturação. (fator $A$ de Zondek). H. M. Fig. n. ${ }^{\circ} 3,6$.

b) fração luteinizante, cuja função é estimular a formação do corpo anarelo ou o desenvolvimento do tecido intersticial dos testículos $\mathrm{E}^{\prime}$ o hormonio de luteinização. (fator $B$ de ZoNDEK). H. L. Fig. n. ${ }^{\circ}$ 3, 7

Segundo Colombo, Fabião (14) e outros existe um único fator gonadotrópico. A ação diversa dependeria de sua concentração.

$20^{\circ}$ - Gonadotropínas extra-hipofisárias - São constituidas por dois fatores:

a) Gonadotropína corionica ou gravidica humana - Corresponde ao Prolan de Aschein-Zondek. Essas substâncias, elaboradas pela placenta, são encontradas na urina de mulher gravida e apresentam propriedades semelhantes ao fator $\mathrm{B}$ hipofisário, tendo ação predominante sôbre o desenvolvimento do tecido intersticial do testículo. (Fig. n. ${ }^{\circ} 3,8$ ).

b) Gonadotropína do sôro equino - São substâncias encontradas no sôro da egua premhe ,aproximando-se pelas suas propriedades ao fator A hipofisário. A ação que predomina é foliculinizante. Fig. n. 3, 9. Portanto, em relação aos ovários o fator A hipofisário e a gonadotropína equina teriam ação foliculo-estimulante (A. F. E.). O fator B hipofisário e a gonatropina corionica, teriam ação luteinizante (A. L.).

Os hormônios gonadotrópicos extra-hipofisários não são identicos aos hiposifários, não os substituem plenamente. Porém, em virtude das dificuldades técnicas que se nos deparam na obtenção e conservação dos hormônios gonadotropicos hipofisários, na prática, quasi que nos limitamos ao emprego dos extra-hipofisários.

Segundo Selye (15) é provavel que a ação do $H$. L. na formação do corpo luteo dependa da sensibilização previa do ovário por um principio hipofisário ausente na urina gravidica cuja elaboração seria estimulada pelo próprio H. L. Provavelmente esse principio é o H. M. 
A aplicação desses hormonios em clínica, fundamenta-se no conhecimento de suas ações fisiológicas. Assim, como são substâncias estimulantes das glândulas sexuais, empregar-se-á nos casos de hipogonadismo masculino ou feminino, consequentes ao deficit hipofisário. $\mathrm{Na}$ prática emprega-se com resultados satisfatórios a gonadotropina corionica no tratamento de certas formas de hipogonadismo secundário como no caso de criptorquidismo. Sob a ação desse hormonio poderse-á observar muitas vezes a descida dos testículos. Quando se nos apresentam estados de hipogenitalismo consequentes ao deficit dos hormonio sexuais, poder-se-á tentar estimular a elaboração desses hormonios empregando-se as substâncias gona'dotrópicas. Entretanto, na prática, os resultados obtidos não são convincentes, pois o efeito é apenas temporário. Os preparados hipofisários têm sido empregados no tratamento de determinados disturbios menstruais devidos ao hipopituitarismo, porém, segundo a maioria dos ginecologistas, os resultados têm sido inseguros. Nestes casos, póde-se estimular a hipófise empregando-se processos fisioterapicos ou biológicos (transfusão de sangue de mulher grávida, dosando-se préviamente as substancias goñadotrópicas).

\section{LÓBO POSTERIOR DA HIPÓFISE}

Injetando-se extrato de lóbo posterior da hipófise no organismo vamos observar uma série de efeitos que encontram aplicações em clínica. Assim, ele determina aumento da pressão arterial, contração dos musculos lisos, expansão dos melanóforos, hiperglicemia, diminuição da diurese, etc. Desse extrato foram isoladas duas fracções;

a) Pitressina ou vaso-pressina ou tonefina ou Beta-hipofamina. Essa substância eleva o tonus da musculatura lisa das arteriodas, (fator hipertensivo) do intestino, do tracto urinário, tendo ainda ação anti-diurética. Fig. n. $.^{\circ}, 10 \mathrm{~b}$.

b) Pitocina ou Oxitocina ou Alfa-hipofamina. A ação dessa substância sôbre o útero é muito intensa daí ser conhecida como fator oxitocico. Fig. n. ${ }^{\circ} 3$ 10a. Em clínica são multiplas as aplicações dos extratos hipofisários. Eles são inativos por via oral, devendo-se ministrá-los por via parenteral ou através da mucosa nasal. Alguns produtos encerram extrato total de hipófise, outros o fator hipertensivo e, finalmente, outros o fator oxitocico.

Procurou-se empregar o extrato total ou o fator hipertensivo no tratamento do colapso periférico. Os trabalhos de Grollman (16) autorizam a proscrever tal indicação terapêutica em virtude de sua ação depressora sobre o coração, provocando constricção das coronárias. Aliás, em individuos sensibilidados as injeções desse medicamento pódem provocar o próprio colapso periferico, acreditando-se que essa reação dependa, além da vaso-constricção coronária, da inhibição dos processos de oxidação ao nivel dos tecidos. Na prá- 
tica emprega-se essa substância no tratamento da distenção abdominal provocada por gazes, para aumentar a nitidez das radiografias abdominais (ação sobre a musculatura lisa do intestino), no tratamento sintomático do diabetes insipidus, etc. Neste último caso dá bom resultados o extrato em pó de lóbo posterior por aspiração nasal.

O fator oxitocico tem também aplicações terapêuticas. Assim ele é empregado para estimular a contração do utero em certas fases do trabalho de parto e controlar a metrorragia post-parto dos uteros atonicos. $O$ emprego dessa substância tem indicações precisas porém limitadas em obstetricia.

$$
* * *
$$

Mussio Fournier (17) e colaboradores têm empregado com exito o hormonio melanóforo no tratamento do vitiligo.

\section{HORMONIOS SEXUAIS FEMENINOS}

Os ovários elaboram pelo menos dois grupos de hormônios sexuais femininos:

a) hormônios estrogênicos ;

b) hormônio de corpo luteo.

Ainda é questão controversa a gênese normal do hormônio estrogênico ovariano, pois alguns atribuem-na as celulas da teca interna do foliculo, outros às células intersticiais.

\section{HORMONIOS ESTROGENICOS}

São assim denominados em virtude de sua ação fundamental que é provocar o estro em animais castrados. Aliás, o test de AleenDorsy que as identifica é baseado nesta ação. Em 1927 Aschein e ZONDEK verificaram que as urinas de mulher gravida e das eguas prenhes são particularmente ricas em substancias estrogênicas. RUteNANDT, (19) em 1929, conseguiu isolar a primeira substância estrogênica, denominada estrona ou foliculina, sendo inicialmente considerada com o verdadeiro hormônio folicular. Posteriormente, SchWENk e HiLDEbrandt (20) conseguiram reduzir a molecula de estrona, preparando assim o estradiol ou di-hidrofoliculina, que é a substancia estrogênica maị poderosa. Essa substancia existe normalmente no liquido folicular, sendo extraida dos ovários de porcas por Dorsy (21). Ela tambem é encontrada na urina gravídica. Hidrogenando-se o estradiol obtem-se o estriol que já foi tambem extraido da urina gravidica. 
$\mathrm{Na}$ prática, emprega-se de preferência, o estradiol sob a forma de benzoato de estradiol, pois a molécula esterificada torna-se ainda mais ativa. A grande fonte dessas substâncias é a urina gravidica, acreditando-se que a placenta tenha capacidade de elaborá-las. A aplicação inadequada dos termos estrona, estradiol e estriol tem determinado uma confusão fundamental de conceitos, o que acarreta. consequentemente conclusões erroneas. Devemos seguir as normas aconselhadas por Thales Martins (22) empregando-se a expressão "hormônio folicular ou estrina", (Fig. n. ${ }^{\circ} 3,11$ ) para se designar genericamente o hormonio folicular ovariano no seu sentido biologico. Quando a designação tem caráter especifico, empregar-se-ão os termos estradiol e estrona, dizendo-se portanto que o hormonio folicular se encontra sob a forma de estradiol nos ovários; de estrona, na urina. Da urina de egua gravida foram extraidas as seguintes substancias estrogênicas: equilina, equilenina e dihidroequilenina.

São multiplas as aplicações dos hormonios estrogênicos em clínica. Porém, dever-se-á ter sempre o cuidado de administrá-las em dóses e ocasiões determinadas, procurando-se imitar o mecanismo fisiológico. Daí a necessidade imprescindivel do conhecimento prévio da fisiopatologia sexual. Em virtude do carácter elementar desta. palestra, nos limitaremos a indicar apenas os efeitos fundamentais. dessas substâncias. Sabemos que o "hormônio folicular" (Fig. n.. 3 , 11) mantem na fase ante-pubere, a nutrição e o desenvolvimento dos caracteres sexuais. Por outro lado, como vimos, a sua elaboração está subordinada à hipófise. Sobrevindo a puberdade, a sua ação torna-se ainda mais complexa. $O$ foliculo chega a se romper libertando o óvulo e no seu leito forma-se à custa das células da. teca interna uma nova entidade endocrínica: o corpo amarelo. Paralelamente, o útero passa a sofrer modificações ciclicas de evolução eregressão na dependência funcional dos ovários. Sob a ação do hormônio folicular, todo o aparelho sexual feminino sofre modificações. ciclicas. Assim, verificar-se-á proliferação do endométrio, (Fig. 3, 11), maior vascularização e hipertrofia do útero, crescimento e permeabilização das trompas, espessamento e proliferação do epitélio da vagina, etc. Atua ainda sobre o seio provocando-lhe o desenvolvimento como já foi assinalado.

Como se póde prever, o hormônio folicular ou estrina, tem inumeras aplicações. Assim, poder-se-á empregá-lo no tratamento de: certos disturbios menstruais endocrinicos (amenorréia; dismenorréias, oligo-menorréia, etc., etc.), perturbações da menopause, hipoplasia genital, vulvaginites gonocócicas perturbações tróficas genitais, nos. casos de hiperplasia dolorosa prémenstrual dos seios, certas formas de esterilidade feminina, etc. Favorece ainda o desenvolvimento dos. prematuros. 
Mussio e Fournier (17) e colaboradores têm utilizado o hormônio folicular por via cutânea, no tratamento do acne juvenll e da hipertricose com excelentes resultados. Entre nós, o problema foi estudado por Gonzalez Torres (18) que confirmou as pesquizas dos autores uruguaios.
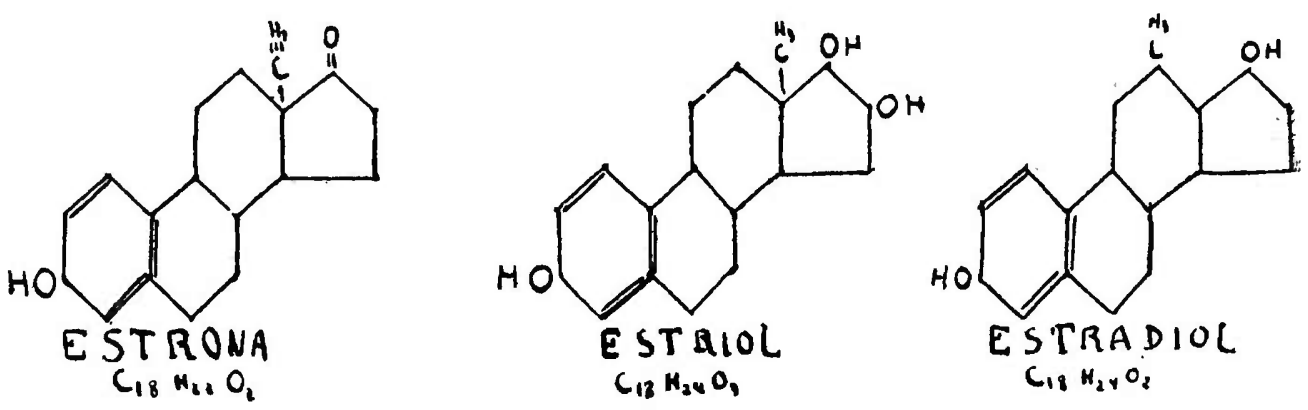

FIG. 1

Estandardizaçâo - A unidade internacional de estrona corresponde a 0,1 milesimo de miligramo de um preparado estandard cristalizado. Portanto, $1 \mathrm{mgrs}=10.000$ unidades internacionais. A unidade internacional benzoica corresponde a 0,1 milesimo de miligramo de um preparado cristalizado de benzoato de estradiol. Logo $1 \mathrm{mgr}=10.000$ U. B. I.

Substâncias estrogênicas artificiais - Vários pesquizadores têm descrito inumeras substâncias orgânicas artificiais com atividade: estrogênica. Com efeito, verificou-se que um derivado estibeno, denominado dietil-estilboestrol ou simplesmente estilboestrol, quando injetado em animais castrados ou femeas jovens provocam-lhes o estro.

Ao lado do estilboestrol foram descritas inumeras substâncias. com propriedades estrogênicas (hexestrol, trifenil-cloro-etileno,, etc.).

Procurou-se então substituir na clínica o hormônio folicular portais substâncias que apresentariam as vantagens do preço menos elevado e de maior atividade por via oral. Mas, as opinióes dos clínicos ainda não são uniformes. Assim, acham alguns que por emquanto devemos proscrevê-las do arsenal terapêutico, pois, com frequência, provocam fenomenos tóxicos, como perturbações gastrointestinais (náuseas, vómitos, cefaléias, diarréias, cólicas, etc.), pros-tração etc.

No entanto, clínicos de nomeada como Castex e SchteinGART, (23) em recente trabalho afirmam: "os resultados terapêuticos do stilboestrol e de seus derivados são semelhantes aos obtidos. 
com a foliculina, com a desvantagem de trazer, conforme as doses empregadas, perturbações gastro-intestinais, mais ou menos acentuadas e em proporção com as doses (30 a $60 \%$ com 5 mgr. e $20 \%$. com 1 mgr.). Essas perturbações não trazem aparentemente consequência alguma e são passageiras"

Dodds, Golbert, Lawson e Robinson. (24) verificaram que o dietilestilboestrol é estrogênico na dose de 0,004 mgr. dado subcutaneamente em veículo oleoso e na dose de 0,001 dado por boca. Cada miligramo de dietil-estilboestrol corresponde aproximadamente a 10.000 U. I. de hormonio folicular.

\section{HORMONIOS DO CORPO LUTEO}

Fraenkel, em 1903, conseguiu demonstrar a atividade endocriníca do corpo amarelo, cujo hormônio é identificado pelo test descoberto por Allen e Corner (1925). Em 1934 foi ele obtido cristalizado, sendo a sua extrutura molecular determinada por Slotta (25), que o denominou luteosterona. Posteriormente Allen conseguiu tambem isolar esse hormônio denominando-o progestina.

Partindo da estigmasterina (substancia encontrada no oleo da fava do feijão soja) e do pregnandiol (alcool inativo encontrado na urina da mulher gravida) FERNHOLz e ButenandT (26) sintetisam-no em -934.

KaUFFMAN demonstrou que os hormonios sintéticos tinham ação idêntica a dos naturais.

No 3. ${ }^{\circ}$ Coñgresso para padronização internacional de Hormonios Sexuais, reunido em Londres (1935), resolveu-se unificar a nomenclatura do hormonio do corpo amarelo, creando-se o termo progesterona ("proge" de progestina (ALLEN) e "sterona" de luteosterona (SLOTTA).

Quando às suas propriedades fisiologicas (Fig. n. ${ }^{\circ} 3,12$ ) sabemos que a fundamental é transformar o endometrio proliferado sob a ação da estrina em endometrio pré-gravidico (fase de secreção) preparando-o para receber o ovo que ainda é por êle protegido (corpo luteo gravidico), daí a sua grande importância na manutenção da gravidez.

Loeb (27) e outros pesquizadores demonstraram que a retirada dos ovários ou do corpo luteo de uma cobaia, após a fecundação, impede a fixação do ovo. HISAW (28) em 1930 mostrou a ação dos extratos luteinicos inhibindo a contraúibilidade do utero. Acredita-se que a progesterona é a responsavel pela hipo-tonicidade uterina observada durante a gravidez. Sabemos que ela inhibe a ovulação e atua concomitantemente com o hormonio folicular, no desenvolvimento das mamas. $E^{\prime}$ interessante registrar a opinião de vá- 
rios endocrinologistas modernos que admitem a secreção de hormônio folicular pelo próprio corpo amarelo.

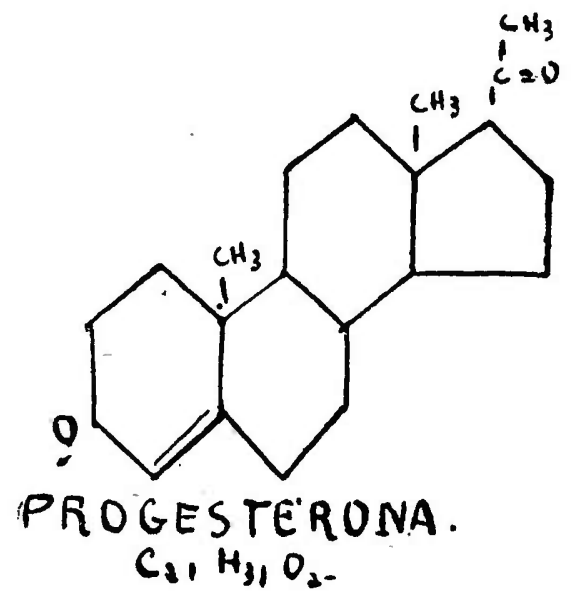

$\mathrm{C}_{2}, \mathrm{H}_{31} \mathrm{O}_{2-}$

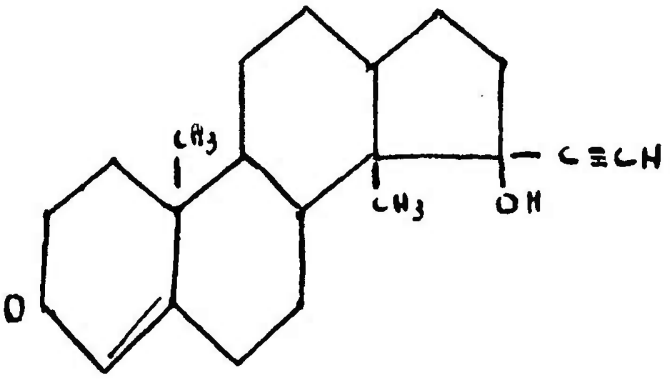

PREGNENINOLONA.

$\mathrm{C}_{2}, \mathrm{H}_{26} \mathrm{O}_{2}$

FIG. 2

Recentemente preparou-se um derivado da testosterona denominado pregeninolona, cuja ação sôbre o organismo assemelha-se à da progesterona. Esta substância bi-sexual, pois tem a atividade androgênica e progestecional, apresenta particularidade interessante de agir sôbre o utero, independentemente do hormonio folicular, sendo ainda ativa por via oral. Ela tem sido empregada em clinica com resultados promissores. A progesterona é utilizada no tratamento do abortamento habitual de fundo endocrinico, tendo tambem ação nitida sôbre os vómitos da gravidez.

Estandardização - A unidade internacional de progesterona corresponde a $1 \mathrm{mgr}$. de hormonio cristalizado.

\section{HORMONIOS SEXUAIS MASCULINOS}

A existência da função endocriníca testicular ficou definitivamente provada quando a experimentação veiu demonstrar os profunidos transtornos somato-psíquicos observados em consequência da castração. Realmente, quando ela é executada no periodo ante-puberal, verificar-se-á inhibição do aparecimento ou do desenvolvimento dos caracteres sexuais somato-psíquicos peculiares ao sexo masculino, . ficando o organismo feminilizado. Após a puberdade ela determina regressão de alguns caracteres sexuais somaticos primários, sendo variavel a repercussão sobre o psíquismo (modificações da libido). A administração dos principios ativos testiculares corrige tais perturbações. Portanto, os hormonios testiculares ou substâncias androgenicias são responsaveis pelo desenvolvimento e conservação dos caracteres sexuais masculinos, sendo elaborados pelas células intersticiais de LEYDIG. 
Foi Butenandt (29) quem isolou da urina do homem as primeiras substâncias androgenicas que foram denominadas androsterona e dehidroandosterona.

Posteriormente DAvid e LAQUER (30) conseguiram extrair dos. extratos testiculares uma nova substancia androgenica muito mais ativa que as já descritas, sendo denominada testosterona. Em seguida, ela foi sintetisada por RUZICKA.

Varios pesquizadores (Roussel, Girard, Wettstein (31) e outros) verificaram que a esterificação da testosterona pelo ácido. proprionico tornava-a ainda mais ativa, creando-se assim o propionato de testosterona, sendo sob essa forma que se emprega. em clínica o hormonio testicular.

O proprionato de testosterona constitue atualmente a substancia. androgênica mais poderosa, sendo empregado geralmente sob a forma de injeções intra-musculares em veículo oleoso, podendo-se, no entanto, utiliza-lo ainda sob a forma de implantação subcutânea out em unções.

Recentemente preparou-se um derivado da testosterona, denominado metiltestosterona que apresenta a particularidade interessante de ser bastante ativo quando empregado por via oral.

As indicações clinicas do hormonio testicular derivam do conhecimento de sua ação fisiológica.

Portanto, em certos casos de hipo-função testicular, não sendo. possivel a terapêutica estimulante hipofisária pelas substâncias gonadotrópicas, torna-se mistér recorrer à ação supletiva do hormônio testicular. Assim, será êle empregado nos casos de eunocoidismo por hipogonadismo primário (castração pré-puberal), cirurgica ou acidental ou secundária - (infecções, avitaminoses, perturbações hipotalâmicas, etc.), observando-se após o emprego de altas doses de hormônio durante certo tempo, não só o desaparecimento da efeminização pela aparição dos caracteres sexuais somato-psiquicos peculiares ao sexo masculino, como tambem aumento do peso corporal e do metabolismo básico. Nos casos em que a causa do eunocoidismo é o hipogonadismo secundário, como em certos casos de criptoquidismo, o tratamento fundamental é clinico (empregando-se substâncias gonadotropicas) ou cirurgico, podendo-se associar subsidiaramente em certos casos o hormônio testicular.

Ainda é questão controversa o seu emprego na hipertrofia prostatica benigna. Com bons resultados tem sido empregado para corrigir as perturbações que sóem acompanhhar o climatério masculino.

Baseando-se em sua ação sobreo lobo anterior da hipofise, inhibindo a formação de substancias gonadotrópicas, Fig. 3, 13x pro- 
curou-se empregar o hormônio testicular em ginecologia, constituindo-se assim a hormônoterapia heterologa que tem dado excelentes resultados na prática.

Segundo os MAzer, (32) o propionato de testosterona em grandes doses (30 mgr por dia), inhibe muito nitidamente a função gonadotrópica-hipofisária $\mathrm{Em}$ doses menores atua diretamente sobre o utero. Daí o seu emprego no tratamento das metrorragias funcionais. Segundo Colombo (45) a testosterona não inibe o fator gonadotrópico, mas neutraliza a estrina em circulação, nivelando a curva hormônal o que faz cessar a hemorragia.

TuRPaUlt (33) observou a regressão da miomatose uterina sob a ação do propionato de testosterona. Essa observação foi confirmada por vários autores que passaram a emprega-lo no preparo pre-operatorio das enfermas miomatosas, mormente naquelas que apresentavam hemorragias.

Entre nós, Motтa Maia (34) tem empregado o propionato de testosterona com sucesso no tratamento das perturbações da menopause e no preparo pré-operatório das miomatosas.

Estandardização - E' feito pelo uso do hormonio em miligramas. 1 miligramo de testosterona equivale a $50 \mathrm{U}$. I., sendo que uma U. I. é igual a $1 / 10$ de miligrama de androsterona cristalizada.

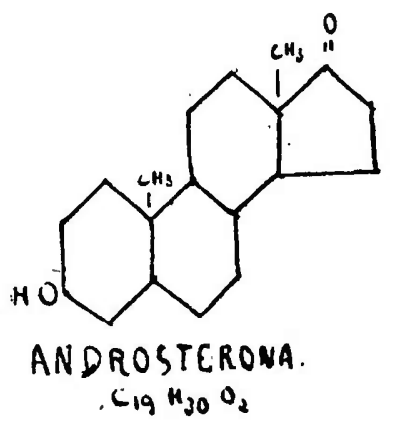

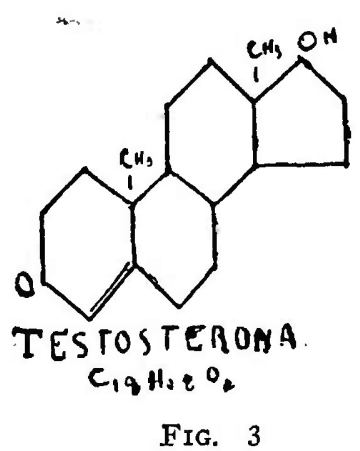

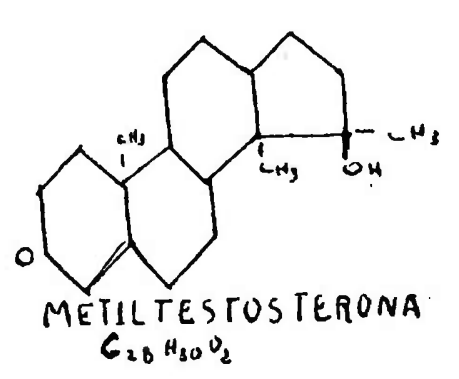

\section{GLANDULAS SUPRARRENAIS}

Encontra-se na glandulas suprarrenais uma parte central, medular de origem ectodermica e outra periférica, cortex, de origem mesodermica. Existe paralelamente uma dualidade funcional, interessando-se no momento, principalmente, o estudo das funções dos hormonios corticais.

Em 1856, BROWN-SÉQUARD demonstrou que as glândulas suprarrenais são indispensaveis à vida. Posteriormente TAKamine e AldRICH isolaram da parte medular da suprarrenal uma substância que foi denominada adrenalina. Esta é a droga simpaticomimétrica por excelência. Em seguida, verificou-se que essa substância não conseguia manter a vida dos animais epinefrectomizados. Atribuiu-se en- 
tão à cortex a propriedade demanter a vida desses animais. $\mathrm{E}$, realmente, em 1927, Stewart e Rogof (35) conseguiram preparar extratos de cortex e suprarrenal capazes de manter a vida de animaisdescapsulizados. Posteriormente, Hartimann, Swingle e PfiffNER (1931) (36) obtiveram um extrato de suprarrenal isento de adrenalina, capaz de corrigir a insuficiencia da glandula. Em 1934 KeNDALL (37) conseguiu extrair do extrato cortico-suprarrenal, uma. substância que foi denominada cortina, tendo por fórmula empirica $\mathrm{C}_{19-20} \mathrm{H}_{28-30} \mathrm{O}_{5}$. Essa substância corrige a insuficiencia suprarrenal. Varios autores conseguiram ulteriormente isolar dos extratos: corticais, várias substancias cristalinas do grupo esterólico, susceptiveis de substituir eficazmente o tecido cortical. Assim, em 1936, Reichstein, (38) na Suiça, conseguiu isolar da cortex uma substância bem ativa, denominada corticosterona. Posteriormente, REICHSTEIN e STEIGERT (39) isolaram outra substancia da cortex mais ativa que a precedente que foi denominada desoxicorticosterona.

Essas substâncias, como os hormônios sexuais, pertencem ao: mesmo grupo esterólico.

Em 1938, conseguiu-se sintetizar a desoxicorticosterona sendo empregada na prática, sob a forma de acetato de desoxicosticosterona. Essas substancias são descritas como hormonios corticais, mas na verdade acredita-se que elas sejam apenas produtos de degradação do verdadeiro hormônio.

$\mathrm{Na}$ prática empregam-se os extratos de cortex ou as substâncias descritas, principalmente o acetato de desoxicosticosterona que é a forma mais ativa. Os extratos corticais oferecem algumas desvantagens como necessidade de grandes doses, dificuldades na padronização, etc. Essas substancias são empregadas em clínica no: tratamento da insuficiencia suprarrenal (Mal de Addison, addisonismos, moléstias infecciosas, intoxicações, queimaduras, choque traumático, etc., etc.). $\mathrm{Em}$ individuos sensiveis elas podem provocar fenomenos toxicos algumas vezes mortais. Daí a necessidade de usa-las com cuidado, procurando-se evitar alguns acidentes como choque hipoglicemico, hipertensão arterial, insuficiencia cardíaca congestiva, edema pulmonar, etc.

A estandardização é feita em miligramas de peso do hormonio.
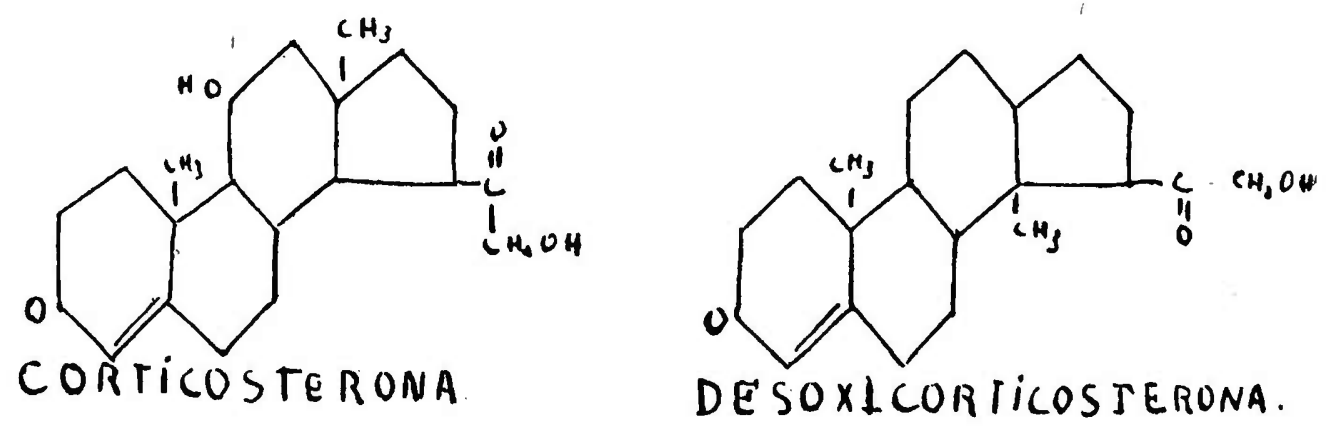


\section{TIREOIDE}

Quando as funções da tireóide tornam-se insuficientes, todos os. tecidos sofrem, o que determina no organismo uma série de perturbações psico-somática caracteriticas. Poder-se-á corrigir tais pertur-bações pela administração de extratos de glandula tiroide (denominados simplesmente "tireóide") ou então de tiroxina, que é um. principio ativo da glândula. Esta é uma forma de hormônoterapia. supletiva.

No extrato glandular, além da tirosina, que é um amino-acido. fundamental do hormonio tiroidiano, encontramos várias substâncias. iodadas: diiodotirosina, tiroxina e tireoglobina. Acredita-se que a tiroxina e diiodotirosina se combinam para formar uma molecula complexa de iodotiroglobulina que seria o verdadeiro hormônio tiroidiano. Dessas substâncias, a que apresenta maior interesse na prática é a. tiroxina que foi isolada da tiróide por Kendall (40) em 1914.

Em seguida, foi ela sintetizada por HaRington, (41) tendo ação: semelhante a da "tiroide" Empregam-se "tiróide" ou tiroxina para. suprir o deficit funcional da glândula. Entretanto, devemos assinalar que a maioria dos clinicos prefere a "tireoide", pois a tiroxina, apesar de apresentar padronização mais perfeita, não dá resul-tados tão satisfatorios na prática.

E' interessante notar que a "tiróide" não é inativada pelos fermentos digestivos, podendo-se ministrá-la por via oral.

Essas substâncias foram tambem empregadas no tratamento decertos casos de obesidade. Dever-se-á, porém, ministrá-las com. grande cuidado, controlando-se o estado geral dos pacientes, (exameclinico repetido, curva de peso, metabolismo basal, etc.), para se evi-. tar os efeitos desagradaveis das super-dosagens.

- A padronização é feita pelo peso do hormônio.
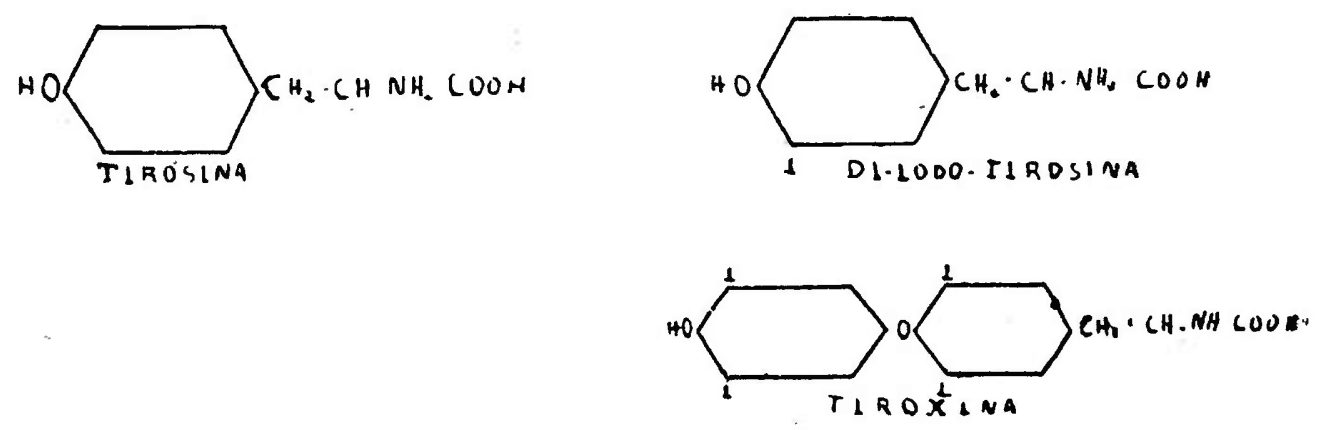

FIG. 5

\section{PARA-TIRÓIDES}

Em 1925, Collip conseguiu preparar um extrato de para-tirói-: des capaz de evitar a tetania e as alterações do equilibrio fosfo-calcio do sangue, dos animais para-tireoprivos. Esse extrato injetado em. 
cães normais determina: hipercalcemia, hipofosfatemia e aumento da eliminação urinaria do calcio e fosforo. Pois bem, nesse extrato encontra-se o paratôrmonio ou hormônio das para-tiroides, que ainda não foi obtido completamente purificado. Apesar de sua ação nitida experimental, na clínica o emprego do paratormônio tem dado resultados um tanto precários. A sua dosagem é dificil e caso se empregue dose excessiva aparecem fenómenos tóxicos. $O$ seu uso repetido provoca no organismo a formação de anti-hormonios, que tornam inativas as doses subsequentes. Acredita-se que esse estado de imunidade aparente esteje relacionado às impurezas que sóem acompanhar o preparado. Usa-se essa substância associada ao calcio nos casos de tetania aguda paratireopriva. Nas formas crônicas poder-se-á empregá-lo associado ao calcio, vitamina $\mathrm{D}$ e taquisterina (A. T. 10).

Estandardização - E' baseada na propriedade que tem o hormônio de elevar a calcemia de um cão normal, após a injeção subcutânea. Uma unidade representa 1 centésimo da dóse necessaria para elevar a calcemia de 1 miligrama por cento, 16 a 18 horas após a sua administração.

\section{PÂNCREAS}

Insulina - Entre os hormônios do pâncreas destaca-se pela sua importância pratica a insulina. Este hormônio é elaborado pelas ilhotas de Langherans, sendo de natureza proteica, portanto inativo por via oral.

A indicação precipua da insulina é no tratamento do diabetes. Em virtude da rapidez de sua ação, o que representa inumeros inconvenientes, procurou-se retardar a sua absorção, creando-se assim as insulinas de depósito.

HAGEDORN (42) e colaboradores empregaram uma protamina extraida do esperma do salmão, como retardadora da absorção. Posterior SCOTt e Fischer (43) acrescentaram ao complexo protaminainsulina uma pequena quantidade de zinco, o que reforça a sua ação e conserva o preparado.

A insulina-protamina-zinco (I. P $\quad$ Z.) difunde-se lentamente no organismo diminuindo consequentemente as reações hipoglicemicas e tendo uma ação mais duradoura, apresentando consequentemente a vantagem de reduzir o numero de injeções.

Atualmente com resultados promissores tem se empregado a insulina nativa.

A padronização da insulina é feita em diabéticos. Encontra-se em Londres e Toronto, uma insulina estandar, sendo que $1 \mathrm{mgr}$. corresponde a 22 unidades internacionais (Apud BERTRAND) (44). 1 centimetro cubico do I. P Z corresponde $a *$ unidades de insulina. 


\section{ESQUEMA DAS INTERCORRELAÇÕES HIPOFISE-GLÂNDULAS SEXUAIS}

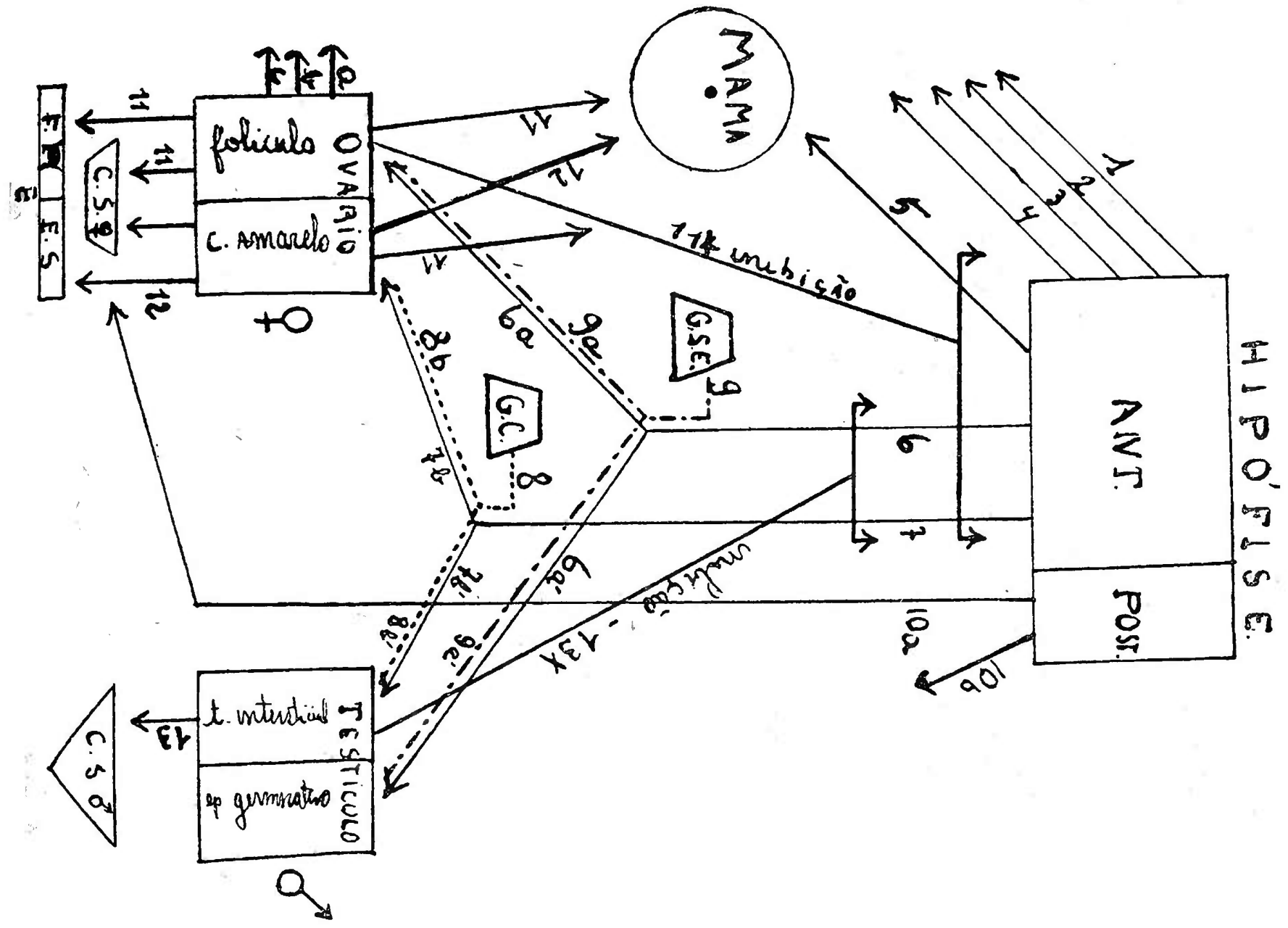

FIG. 0

1, 2, 3 e 4 - hormônios de crescimento tireotrópico, adrenotrópicico e metabólicos..

5 - hormônio lactogênico.

6-7 - hormônios gonadotrópicos (H. G.) - 6 - h. de maturação (H. M.) e 7 - h. de luteinização. (H. L.)

8 - Gonadotropina corionica.

9 - Gonadotropina do sôro equino.

$10 \mathrm{a}$ - fator oxitocico; $10 \mathrm{~b}$ - fator hipertensivo.

11 - h. folicular ou estrina (H. F.) ; 11a - estrona ; $11 \mathrm{~b}$ - estradiol e 11c estriol. $11 \mathrm{x}$ - inibição de pré-hipófise.

12 - h. do c. amarelo - progesterona.

13 - h. testicular - testosterona $-13 x-$ inibição de pré hipófise.

C. S.: cáracteres sexuais femininos
C. S.:
masculinos
$\mathrm{E}=$ endométrio
F. H. = fase hiperplastica.
F. S. = fase de secreção.

O H. M. (6a) agindo sôbre o ovário determina o desenvolvimento do folículo e a ovulação (mais ou menos no $15 .^{\circ}$ dia do ciclo menstrual). O folículo e o corpo amarelo elaboram o H. F. (11) que determina no endométrio a F. H. Após o $200^{\circ}$ dia do ciclo menstrual o corpo amarelo estimulado 
pelo H. L. passa a elaborar progesterona (12) que vai determinar no endométrio a F. S.

$\mathrm{O}$ H. F. atingindo determinado limite de concentração vai anular biologicamente o H. G. na circulação ou, segundo alguns AA., vai inibir a préhipófise, libertando o ovário do estímulo hipofisário. Os folículos em via de desenvolvimento ficam atrésicos, baixa a concentração da estrina, o que causa a desagregação do endométrio, iniciando--se a mentruação. Quando não há ovulação o ciclo é mono-fásico ou anovular sendo esteril, diagnosticado pelo exame hestologico do endométrio - após curetagem - que demonstrará a falta da F. S. Segundo outros, aumentando a concentração da progesterona ela vai inibir a elaboração do H. L., regride o e, amarelo, e o endométrio pré-gravidico desagrega-se, iniciando-se a menstruação (ciclo ovular, di-fásio). Quando dá fecundação o corpo amarelo pré-gravidico transforma-se em gravidico (ciclo tri-fásico).

\section{REFERENCIAS (*)}

1 - Martins Th. - Rev. Med. Bras. - X:303,2,1941.

2 - Thorn e FIror - J. A. M. A. - 114:2517,1940.

3 - Martins Th. - Rev. Med. Bras. X:303,2,1941.

4 - Apud Torres, G. - Rev. de Med. C.A.O.C. - $24: 18,7,1940$.

5 - Parkes e Rowl.ands - Jour. Phys. 38:305,1937.

6 - Collip, Selye e Thomson - Rev. Biol. $15: 1,1940$.

7 - Apud Grollman - Essentials of Endocrinolngy, p. 13, 1941, Lippincott.

8 - Evans - Res. Clin. Cientif. X:109,6,1941.

9 - SCHAEFFER e ENGElbach - Endocrin. $18: 387,1934$.

10, 11 e 12 - Apud Rrvorre - Les acquisitons Nouvelles de L'Endocrinologis. pgs. 165 e' 241 - 1937. Masson Cie.

13 - Stewart e Pratt - Endocrin. 25:347,1939.

14 - Fabião e Lago - Rev. Med. Bras. X :189,1,1941.

15 - Selye - Res. Clin. Cient. X :217,6,1941.

16 - Grollman - Ob. cit. pg. 69.

17 - Mussio Founier - Res. Clin. Cientif. X:177,6,1941.

$18-$ Torres, G. - Arq. Biol. XXV:115,6,1941.

19, 2021 - Apud Martins Th. - Rev. Med. Bras. VIII:163,2,1940.

22 - Martins Th. - Rev. Med. Bras. VIII :163,2,1940.

23 - Castex e Schteingart - Res. Clin. Cientif. X:213,6,1941.

24 - Apud Barbosa Correia - Rev. Clin. S. Paulo VI :15,1,1939.

25, 26 - Slotta - An. Bras. Ginec. $11: 1,4,1941$.

27, 28 - Apud RIvorre - Ob. cit. pg. 144

29, 30 - Apud Martins Th. - Rev. Med. Bràs. VIII :161,2,1940.

31 - Apud Rivorre - Ob. cit. pg. 191.

32 - CH. e M. MAZER - Endocrin. 24:599,5,1939.

33 - Apud Motta Maia - Cult. Méd. II :262,10,1940.

34 - Motta MaIA - Cult. Med. II :262,10,1940.

35, 36 - Apud Rrvorre - Ob. cit. pg. 83.

$37-$ Kendall - J.A.M.A. $105: 1486,1935$.

38 - Apud RIvoIre - Ob. cit. pg. 83.

39 - Reichstein e Steiger - Helv. Chim. Act. 20:917,1937.

40, 41 - Apud GrollmanN - Ob. cit. p. 122.

42 - Hagedom, Jessen, Kraurup e Wodstrup - J.A.M.A. $106: 177,1936$.

43 - Apud Macedo R. - Rev. de Med. C.A.O.C. $24: 27,8,1940$.

44 - Beltram - Diabetes - p. 96 - 1939. Melhoramentos.

45 - Colombo - Rev. Med. Bras. - VIII :183,2,1940.

Rev. Med. Bras. - VIII: 41,1,1940.

(*) Em se tratando de uma simples palestrax de divulgaçāo achamos despropositada a apresentação de enorme biblografia referente ao assunto. 


\section{$\mathbf{N} \boldsymbol{O}$
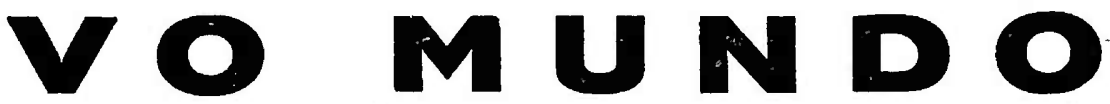

COMPANHIA DE SEGUROS TERRESTES, MARITIMOS

E GARANTIA DE ALUGUËIS E COMPANHIA DE SEGUROS DE ACIDENTES DO TRABALHO

AGÊNCIA GERAL PARA O ESTADO DE SÃO PAULO :

RUA BOA VISTA, $57-2 .^{\circ}$ ANDAR

Telefone: 2-3099

AMBULATÓRIO :

RUA 3 DE DEZEMBRO, 47

Telefone: $3-4442$

Sede: RUA DO CARMO, 65 (Edificio Próprio)

Telefone: 23-59II - RIO DE JANEIRO

$\star$

REPRESENTANTES EM TODAS AS CIDADES DO INTERIOR $\star$

Agência em Curitiba:

RUA 15 DE NOVEMBRO, 599

Agência em Porto Alegre :

AVENIDA JÚLIO DE CASTILHOS, I33

\section{Centrifugadores Elétricos Americanos para Laboratórios}

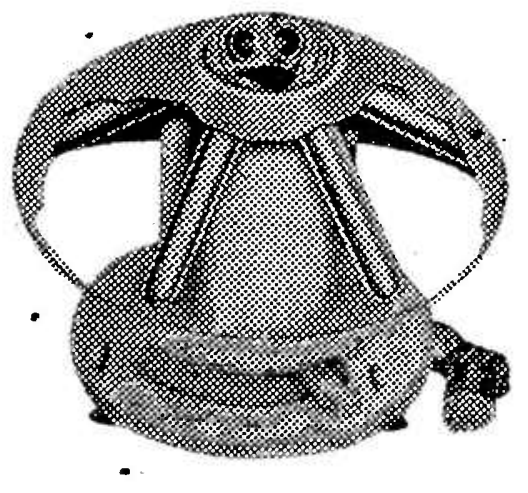

Novos principios: Tubos com ângulo fixo, centro de gravidade baixo, volume reduzidio.

Principais vantagens: sedimentação mais rápida, ausência de vibração, segurança absoluta.

GRANDE SORTIMENTO A PREÇOS baIXOS NA

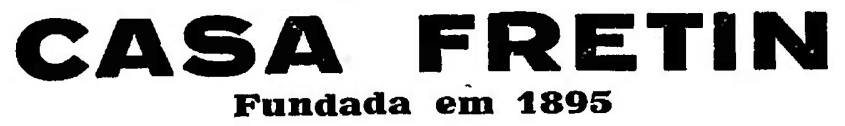

Praca do Patriarca SÃo PAULO - CX. Postal 1273 


\section{Gazes Impregnadas}

GAZES PARA TAMPONAGEM o material indispensavel nas intervenções cirurgicas.

GAZES esterilisada's, hidrófilas, gomadas, impregnadas, laminadas, para tamponagem e outras.

ATADURAS de gaze hidrófila, de cambraia, de gaze gomada, contra queimaduras, rápidas, crepón, tricó, elasticas, gessadas, plasticas, CELLO-FIX e outras.

Fabrica de Gazes Medicinaes "CREMER" S. A.

primeira fabrica de gazes medicinais na America do Sul

BLUMENAU

CAIXA POSTAL 80

S. CATARINA

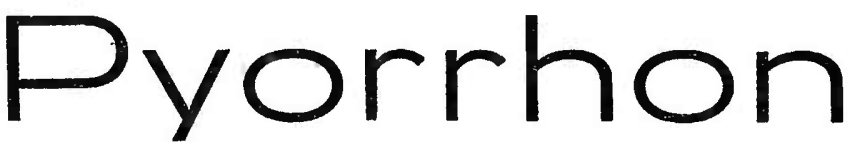

Um medicamento que veio resolver os casos de Gengivites e Pyorhhéa

Receite PYORRHON aos seus clientes

\section{Frio ou Chuva!}

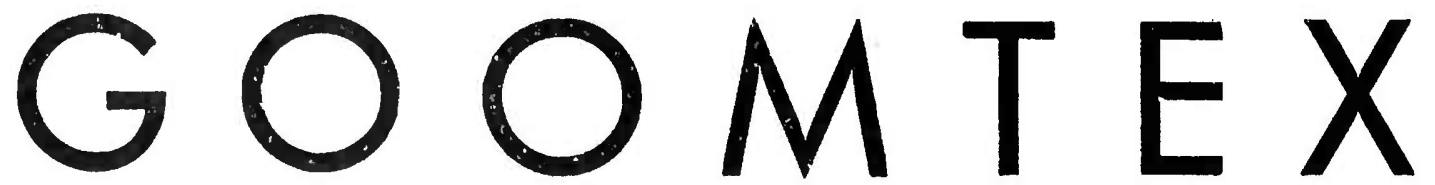

A capa que veste o Brasil 